\title{
SDO: Simulador de Doenças Oculares
}

\author{
Rafael Avilar Sá \\ rfl.avilar@outlook.com \\ Instituto UFC Virtual \\ Fortaleza, Ceará \\ Windson Viana \\ Universidade Federal do Ceará \\ Fortaleza, Ceará, Brasil \\ windson@virtual.ufc.br
}

\author{
Agebson Façanha \\ Instituto Federal do Ceará \\ Fortaleza, Ceará, Brasil \\ agebson@ifce.edu.br
}

\author{
George A. M. Gomes \\ Universidade Federal do Ceará \\ Fortaleza, Ceará, Brasil \\ george@virtual.ufc.br
}

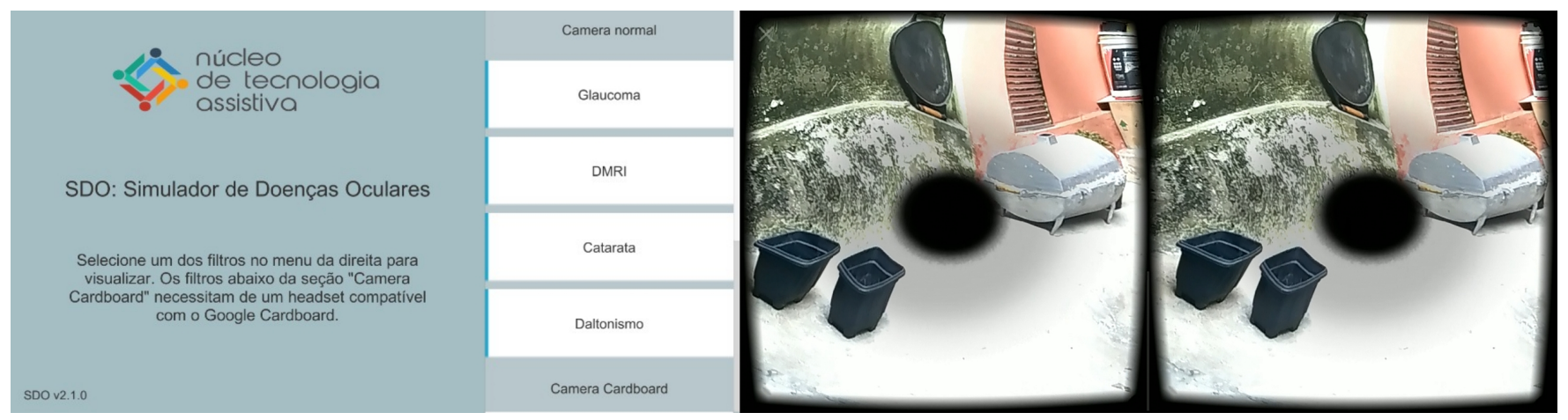

Figura 1: Simulador de doenças oculares e seu Modo Imersão com o Google Cardboard

\begin{abstract}
In Brazil, 2010, more than 6.5 million people claimed to have some visual impairments. Software and Web accessibility are becoming first-class requirements to promote a better relationship between those people with visual impairments and the internet as well as with new digital technologies. However, designers and software developers need more empathy and knowledge about these groups' issues to implement accessibility features into their digital products correctly (e.g., apps, games, Web sites). This paper has the goal of developing an eye disease simulator (SDO - Simulador de Doenças Oculares) for mobile devices, which could help better teach and illustrate the effects of these diseases. First, we investigated how to simulate the symptoms of each disease better and have developed the SDO mobile application. SDO is capable of both simulating different types of Colorblindness as well as other more dangerous eye illnesses (i.e. Glaucoma, cataracts). The current version also supports the use of Head-Mounted Displays to increase user immersion.
\end{abstract}

\section{KEYWORDS}

eye diseases, augmented reality, mobile development, digital image processing

In: XIX Workshop de Ferramentas e Aplicações (WFA 2020), São Luís, Brasil. Anais Estendidos do Simpósio Brasileiro de Sistemas Multimídia e Web (WebMedia). Porto Alegre: Sociedade Brasileira de Computação, 2020.

(c) 2020 SBC - Sociedade Brasileira de Computação.

ISSN 2596-1683

\section{INTRODUÇÃO}

O aplicativo aqui descrito é uma das tecnologias assistivas desenvolvidas pela parceria entre a Universidade Federal do Ceará (UFC) e o Instituto Federal de Educação, Ciência e Tecnologia do Ceará (IFCE). Seu fomento inicial foi feito pelo $\mathrm{CNPq}^{1}$ em 2016. A versão mais atual do SDO foi evoluída pelo estudante Rafael Avilar ${ }^{2}$ sob a orientação dos pesquisadores Windson Viana ${ }^{3}$, George Gomes ${ }^{4} \mathrm{e}$ Agebson Façanha ${ }^{5}$.

Deficiência, de acordo com a Organização Mundial da Saúde (OMS) [18], pode ser definida como um transtorno que afeta a estrutura corporal de um indivíduo, a sua capacidade de executar alguma tarefa ou ação, e o seu estilo de vida. As deficiências visuais, por exemplo, são caracterizadas pela redução no potencial de visão do indivíduo, não corrigíveis por meios comuns (como óculos ou medicação). No Brasil, elas são as mais encontradas, e de acordo com dados do IBGE em 2010, mais de 6.5 milhões de pessoas $(3.4 \%$ da população) declararam possuir algum tipo de deficiência visual [12]. Outra pesquisa, realizada em 2013 , reporta que $16 \%$ das pessoas com deficiência visual no Brasil são incapazes de realizar tarefas básicas como ir à escola, trabalhar ou brincar devido à severidade da deficiência [11]. Dentre as principais causas de deficiência visual,

\footnotetext{
${ }^{1}$ Projeto de Pesquisa do CNPq, sob concessão ${ }^{\circ} 458825 / 2013-1$, elaborado para execução no âmbito da Chamada MCTI-SECIS/CNPq n 84/2013 - Tecnologia Assistiva, sob o título: Pesquisa e Desenvolvimento de soluções digitais para educação, cultura e interação com sistemas móveis para pessoas com deficiência visual

${ }^{2}$ http://lattes.cnpq.br/7187933272456817

${ }^{3}$ http://lattes.cnpq.br/1744732999336375

${ }^{4}$ http://lattes.cnpq.br/5012576140529904

${ }^{5}$ http://lattes.cnpq.br/6135832725966265
} 
destacam-se erros refrativos não-corrigidos, catarata, Degeneração Macular Relacionada à Idade (DMRI), Retinopatia Diabética (RD) e glaucoma [19].

Em geral, é difícil imaginar como exatamente distúrbios visuais podem influenciar no modo de vida das pessoas com deficiência. Especialmente por afetar a forma com que o indivíduo enxerga o mundo, um dos principais sentidos do corpo e parcialmente responsável pela coleta e processamento de informações. A compreensão mais clara de como os distúrbios afetam o dia a dia pode ser um importante elemento de geração de empatia com as pessoas com deficiência visual. Além disso, é importante para alertar efeitos que doenças não tratadas podem causar na percepção visual de um indivíduo, por exemplo, o Glaucoma que pode ser agravado com a diabetes.

Para designers e desenvolvedores de artefatos digitais (e.g., aplicações móveis, páginas Web) também pode ser complexo imaginar como cada tipo de deficiência visual afeta a percepção das interfaces desses produtos. É possível encontrar na Web gráficos, vídeos ou mesmo apps que exemplificam os efeitos de transtornos visuais de forma simplificada. Por exemplo, o aplicativo ColorBlindness SimulateCorrect ${ }^{6}$, disponível na PlayStore, aplica filtros de cor para simular o daltonismo em imagens ou capturas de tela. Todavia, o uso de tecnologias imersivas podem fornecer conexões ainda mais imersivas e significativas com as experiências decorrentes do uso, como diz Bowman no seu estudo sobre Realidade Virtual (RV) [6].

Dentro deste contexto, esta pesquisa tem como objetivo desenvolver e aplicar tecnologias móveis no processo de geração de empatia sobre os diversos tipos de deficiência visual. Foi desenvolvido um Simulador de Doenças Oculares (SDO). O SDO é uma aplicação móvel de Realidade Aumentada (RA) ${ }^{7}$ que aplica filtros na imagem percebida pela câmera do dispositivo móvel para simular doenças visuais como o Glaucoma, DMRI e três tipos de Daltonismo. A aplicação também pode ser integrada a um HMD (Head-Mounted Device) de baixo custo para aumentar a imersão.

Seu uso tem dois principais focos: I - a compreensão da deficiência visual e II - avaliação de artefatos digitais. No caso I, por exemplo, sua aplicação pode ajudar na prevenção à cegueira, abrangendo aspectos individuais relacionados ao paciente como a compreensão da deficiência visual, educação e adesão do paciente a um tratamento médico proposto (e.g., controle da diabetes). Já no segundo caso, o mesmo aplicativo móvel pode ajudar designers e desenvolvedores a simular como usuários com deficiência visual enxergam suas aplicações. Foi produzido um app e vídeo demonstrativo do simulador ${ }^{8}$.

O restante do artigo está organizado da seguinte forma: na Seção 2, os trabalhos relacionados são apresentados. A metodologia utilizada é descrita na Seção 3. Em seguida, na Seção 4, as funcionalidades do aplicativo SDO são explicadas. As tecnologias utilizadas no desenvolvimento são apresentadas na Seção 5 . A relevância e o impacto social são discutidos na Seção 6. Por último, as próximas

${ }^{6}$ https://play.google.com/store/apps/details?id=com.SeewaldSolutions.

ColorBlindnessSimulator\&hl=pt

${ }^{7}$ Realidade Aumentada pode ser definida como uma variação do conceito de Realidade Virtual [4]. Enquanto RV completamente imerge o usuário em um ambiente virtual fabricado, a RA permite visão e interação com o mundo real, justaposto de elementos virtuais.

${ }^{8}$ https:/drive.google.com/drive/folders/18Ns02tgaWCgOwSVkFDqRO9gEWyMpEII?usp=sharing etapas do projeto são tratadas na Seção 7 e considerações finais na Seção 8.

\section{TRABALHOS RELACIONADOS}

O conceito de um simulador de doenças oculares já foi explorado noutros trabalhos com temáticas, objetivos ou inspirações semelhantes a este. Banks [5], por exemplo, realizou uma investigação sobre métodos de processamento de imagens que poderiam ser utilizados para simular deficiências visuais, resultando na construção de um software que realiza modificações sobre imagens estáticas. Ates [3] propõe um modelo de aplicação de conceitos de Realidade Virtual na construção de um dispositivo que simula complicações visuais, o SIMVIZ, direcionado ao contexto de desenvolvimento de softwares acessíveis, fazendo uso das capacidades mais robustas do Oculus Rift ${ }^{9}$. Já Lewis [16] desenvolveu também um simulador, porém utilizando a Unreal Engine ${ }^{10}$ e o seu kit de Realidade Virtual, com o objetivo de explorar a sua capacidade de realizar uma simulação efetiva de doenças visuais. Outro exemplo mais recente é da pesquisa de Choo et al. [7] que avalia o impacto positivo do Empath-D, um simulador em RV de doenças oculares, na avaliação de interfaces Web.

Este projeto se diferencia dos demais em alguns pontos, especificamente: (1) foco na Realidade Aumentada como tecnologia mediadora da experiência, (2) versão para HMD de baixo custo como o Virtual Box, (3) dois usos possíveis: geração de empatia por meio do entendimento dos efeitos das doenças e seu uso para avaliação de acessibilidade de artefatos digitais e (4) o seu status como software livre feito na Unity Engine ${ }^{11}$.

\section{METODOLOGIA}

O projeto do SDO foi concebido em um workshop de inovação em tecnologias digitais para a saúde (Figura 2). Estudantes, pesquisadores e médicos (e.g.,oftalmologistas) foram convidados para discutir aplicações móveis no contexto da área de saúde. Durante uma seção de brainstorming, o grupo procurou por conceitos de aplicações móveis que poderiam transformar o relacionamento entre médicos e pacientes ou aumentar a retenção de pacientes a tratamentos de prevenção à cegueira. Foi assim que os primeiros requisitos e princípios de design do SDO foram elicitados.

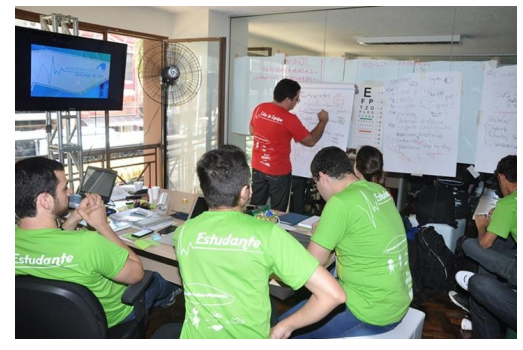

Figura 2: Brainstorming do design do SDO.

Após o workshop, a pesquisa continuou seguindo um processo baseado no Design Centrado no Usuário (User Centered Design,

\footnotetext{
$\overline{{ }^{9} \mathrm{https}: / / \text { www.oculus.com/rift }}$

${ }^{10} \mathrm{https}$ //www.unrealengine.com/en-US/

${ }^{11}$ https://unity.com/
} 
UCD) [1]. O design centrado no usuário foca em tratar do usuário como aspecto central do processo de desenvolvimento, cujos desejos e objetivos devem diretamente influenciar nas escolhas realizadas [1]. Essa abordagem é geralmente baseada em três princípios: (1) Foco inicial nos usuários e tarefas; (2) Pesquisas empíricas; E (3) design iterativo [9]. Seguindo a metodologia, em 2016, foi desenvolvida uma versão inicial do simulador. Na sua primeira iteração, já possuía as seguintes funcionalidades: menu principal, filtros de glaucoma, DMRI e catarata. Também apresentava uma maquete 3D experimental da estrutura interna do olho humano, para ser explorada como ferramenta de aprendizado. No segundo semestre de 2019, iniciou-se um processo de melhoria e extensão do aplicativo. A atualização teve como objetivo primário o desenvolvimento de uma funcionalidade de aumento de imersão, fazendo uso de tecnologia RA com HMDs. Para complementar essa adição, também foi planejado suporte a um controle externo do aplicativo por meio de um joystick Bluetooth, além de uma otimização geral da base de código e adição de um novo filtro (que simula tipos de daltonismo). Devido a esses novos objetivos, o desenvolvimento da funcionalidade de maquete 3D foi pausado.

O projeto é de âmbito acadêmico-social, sem fins comerciais (licensa Open Source CC BY-NC-SA 4.0). A ideia é criar uma ferramenta que possa ajudar a impactar a sociedade de forma positiva, apoiando os esforços da comunidade científica e contribuindo para o avanço do desenvolvimento de tecnologias acessíveis.

\section{FUNCIONALIDADES}

Como mencionado, o aplicativo SDO possui 4 filtros que simulam doenças oculares e uma função para uso por Head-Mounted Devices. A Figura 3 ilustra o conceito por trás do aplicativo. O SDO captura a cena do mundo real por meio da câmera traseira do dispositivo e aplica um filtro na imagem capturada, cujo resultado é renderizado na tela do dispositivo. Essas funcionalidades são descritas em mais detalhes a seguir.
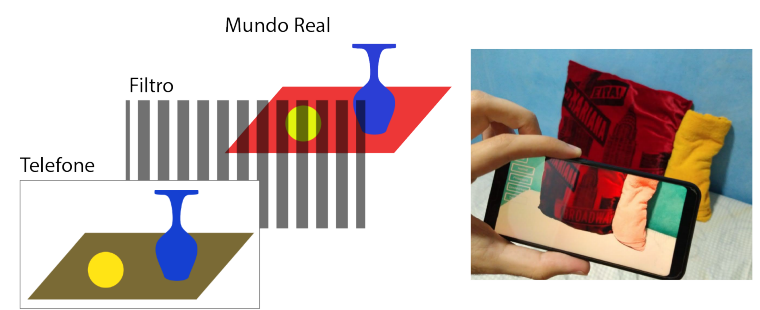

Figura 3: O funcionamento do aplicativo SDO.

\subsection{O Filtro de Glaucoma}

O Glaucoma é uma designação padrão para um grupo de doenças que compartilha de características similares, sendo a variação mais comum definida pela perda de visão periférica que, se não tratada, pode eventualmente resultar em cegueira total [15]. Essa perda de visão induz um efeito de escurecimento da região periférica ao redor do cone central. No simulador, a doença foi replicada com um filtro composto por texturas e mudança de transparência que são reguladas na interface do aplicativo para criar uma simulação dinâmica, que pode representar diferentes estágios da doença.

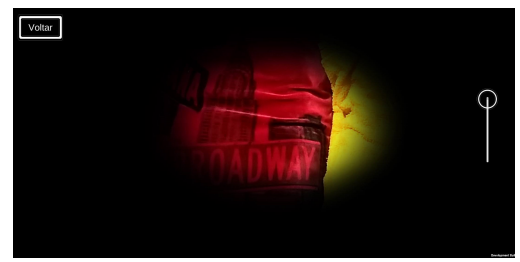

Figura 4: Exemplo da aplicação do filtro de Glaucoma.

\subsection{O Filtro de DMRI}

A Degeneração Macular Relacionada a Idade (DMRI) é uma doença visual comum em indivíduos acima de 50 anos caracterizada pela perda gradual da visão central [2]. Mácula refere a região localizada no centro da parte traseira da retina. Os principais sintomas de degeneração macular relacionada à idade são: visão borrada, redução/manchas no centro da visão, enxergar as cores mais desbotadas, dificuldade de adaptação ao escuro, linhas distorcidas e tortuosas e necessidade de iluminação mais intensa para ler em casos mais avançados [2]. Para a simulação, foram utilizados uma combinação de efeitos diferentes, que em conjunto simulam alguns dos possíveis sintomas da doença.

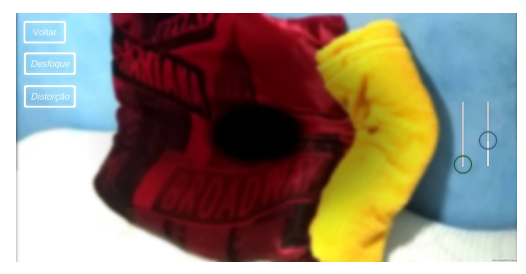

Figura 5: Exemplo da aplicação do filtro de DMRI.

\subsection{O Filtro de Catarata}

A catarata ocorre como uma espécie de lesão localizada no cristalino do olho. Como resultado, o paciente apresenta dificuldade de enxergar com nitidez, como se possuísse uma névoa em frente ao olho [8]. Também pode sofrer visão borrada, imagens duplicadas e sensibilidade à luz [13]. Para representar esse efeito, o filtro faz uso de transparência combinado a luz branca, permitindo aumentar ou diminuir a intensidade da luz.

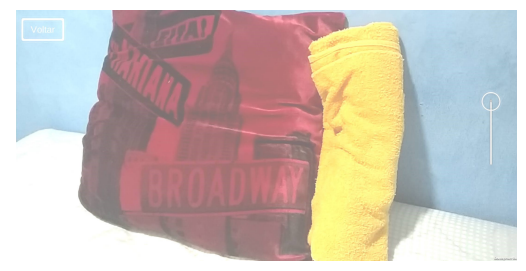

Figura 6: Exemplo da aplicação do filtro de Catarata.

\subsection{O Filtro de Daltonismo}

O daltonismo é caracterizado pela redução da capacidade do indivíduo de enxergar ou diferenciar tons de cores. A condição é dividida 
em alguns tipos, dependendo dos pigmentos afetados, sendo o daltonismo de deficiência vermelho-verde considerado o mais comum [14]. Para a simulação desse efeito, foi utilizado um pacote externo desenvolvido por Ian Pudney [10]. Ele faz uso de shaders ${ }^{12}$ para alterar as cores dos pixels da tela. O simulador possui três possíveis configurações para este filtro: protanopia, deuteranopia e tritanopia.

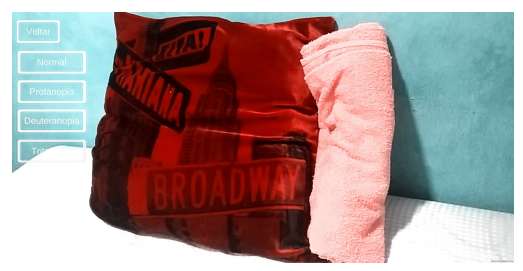

Figura 7: Exemplo do filtro de Daltonismo (tritanopia).

\subsection{Modo Imersão}

O desenvolvimento da funcionalidade de imersão do SDO, referida desde então como Modo Imersão, foi uma das etapas do projeto cujos maiores esforços foram dedicados. Através dele, é possível criar uma espécie de ambiente imersivo para execução de testes ou experimentos de imersão. Como informado anteriormente, cada filtro é composto de uma cena e um script. O Modo Imersão foi implementado através da construção de uma cena adicional, uma versão modificada da original, em que os objetos foram reconfigurados para suportar o uso de HMDs. Ele foi desenvolvido com o Google Cardboard ${ }^{13}$ em mente, ou seja, deve suportar qualquer dispositivo que usa-o como base. No entanto, foi testado apenas no VR Box ${ }^{14}$, uma das opções mais acessíveis encontradas, custando aproximadamente 50 reais incluindo o joystick. Na figura abaixo (Figura 8), é possível visualizar o Modo Imersão com a aplicação do filtro Daltonismo.

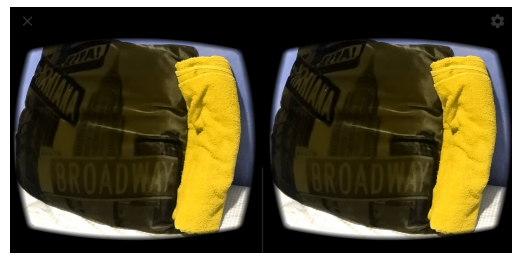

Figura 8: Simulação no Modo Imersão.

\subsection{Controle por Joystick}

O suporte para o joystick foi implementado com a biblioteca de inputs do Unity. O esquema de controle padrão foi programado com base no joystick do VR Box ${ }^{15}$. O objetivo do Joystick é fornecer o controle das interações (mudança de filtros e configurações dos filtros) quando o usuário estiver usando a versão para aumento de imersão na qual o smartphone é embarcado no HMD, ou mesmo para permitir que um usuário utilize o simulador enquanto outro controla.

\footnotetext{
${ }^{12}$ scripts especializados em processamento de imagem

${ }^{13} \mathrm{https} / / /$ arvr.google.com/cardboard/

${ }^{14} \mathrm{https} / / /$ www.aniwaa.com/product/vr-ar/vr-box-2-0/

${ }^{15}$ Manual do joystick do VR Box: http://v3.hamiltonbuhl.com/hammanual/Universal\% 20VR\%20Remote\%20Controller-Manual-d10.pdf
}

\section{DESENVOLVIMENTO}

O aplicativo foi desenvolvido na Unity Engine, versão 2019.2.0f1. Ela é um motor de jogo, ferramenta capaz de abstrair detalhes comuns da implementação de jogos como renderização, física, input e exportação. Todos os scripts relevantes do SDO foram programados em C\#, linguagem padrão de desenvolvimento da Unity Engine. Também foi usado o pacote externo ${ }^{16}$ Colorblind para auxiliar na programação do filtro de daltonismo [10].

Devido ao uso de programação baseada em componentes, os scripts, materiais e cenas do projeto são modulares. A base de códigos é portanto desacoplada e facilita manutenção e modificações futuras. Cada filtro, por exemplo, é representado por um script e uma cena. O script é responsável pela lógica de funcionamento do filtro, e a cena contem o script, o objeto que representa a câmera, a interface e demais recursos necessários para a simulação do efeito. Para testes e debugging, foram utilizados celulares e um aparelho VR Box. O aplicativo foi testado nos seguintes dispositivos: Xiaomi Mi A2 Lite, Moto G5 Plus, Motorola One. O repositório do projeto está disponível na plataforma GitHub ${ }^{17}$.

\section{RELEVÂNCIA E IMPACTO SOCIAL}

A perda da visão, em qualquer nível, leva a uma restrição no desempenho de atividades diárias, o que interfere na independência e autonomia do indivíduo e, por conseguinte, na sua qualidade de vida. O uso de auxílios ópticos, não-ópticos e adaptação do ambiente tem sido uma alternativa para melhorar o desempenho das pessoas com deficiência visual em suas atividades. A visão é um dos cinco sentidos, e é considerada por muitos como o mais precioso. A visão nos dá acesso à maioria das informações sobre o mundo a nossa volta. Compreendendo como cada patologia afeta a visão permite que o tratamento seja individualizado, evitando a generalização do tratamento pela condição visual. Por isso, é muito importante conhecer as doenças oculares que podem acometer o sistema visual, que provocam desde uma discreta diminuição da acuidade visual até a cegueira definitiva.

O SDO entra neste contexto como uma ferramenta para conscientização, suporte educacional e auxílio de testes de acessibilidade. Através de Realidade Aumentada, as principais doenças oculares podem ser simuladas na maioria dos dispositivos Android (mesmo os de baixo custo) com um fator adicional de imersão se combinadas com um HMD. Essas funcionalidades permitem que o paciente compreenda a patologia bem como a implicação visual de cada uma delas, se não tratadas ou em estágios iniciais. Pacientes, assim como cuidadores e familiares podem, com o aplicativo, entender melhor as dificuldades provindas das doenças, e a importância de adesão aos seus respectivos tratamentos.

Este impacto social pode ser percebido durante a amostra do simulador na feira do conhecimento promovida pelo Governo do Estado do Ceará (em 2018 e 2019). Exibido em um stand na mostra de tecnologias assistivas, juntamente com outros aplicativos de acessibilidade do grupo GREaT, a primeira versão do SDO já permitiu que mais de 200 pessoas pudessem experimentar essa tecnologia.

\footnotetext{
${ }^{16}$ Pacotes são abstrações utilizadas pelo motor de jogo que se assemelham a bibliotecas, porém podem ser compostas de diversos recursos diferentes, como materiais, texturas, scripts e arquivos.

${ }^{17}$ https://github.com/rafero1/sdo-public
} 
Embora, nenhuma avaliação rigorosa tenha sido executada com os participantes, era perceptível pelos feedbacks e reações a surpresa dos usuários com os efeitos das doenças. Com isso, espera-se que, nos participantes, o uso do simulador tenha contribuído para a geração de empatia com aqueles que possuem deficiência visual, a partir do entendimento das suas dificuldades diárias. Outro ponto salutar da feira foi embutir nesses usuários a importância de investimento no desenvolvimento destas tecnologias.

Também acreditamos que o SDO poderá impactar no contexto de desenvolvimento de softwares acessíveis. Designers e desenvolvedores podem usar a simulação para melhorar produtos digitais que levem em consideração as particularidades das pessoas com deficiência visual. Além disso, o aplicativo encontra uso em contextos educacionais. Médicos, professores ou profissionais da área de saúde podem utilizar o simulador como um Objeto de Aprendizagem (OA) para melhorar o entendimento sobre os efeitos das doenças nas ações do dia a dia (e.g., leitura, movimentação).

\section{PRÓXIMAS ETAPAS DO PROJETO}

Inicialmente, a versão atualizada do simulador seria exibida na Feira de Conhecimento de 2020 no estado do Ceará. Também, estava previsto seu uso no dia mundial de combate a diabetes em parceria com um grupo de endocrinologistas da cidade de Fortaleza em uma campanha para explicar os efeitos que o Glaucoma pode causar nas pessoas com diabetes sem controle. No entanto, devido às medidas de quarentena para o combate à proliferação da COVID-19, estes planos foram adiados para 2021.

Apesar disso, foi planejada uma lista de atividades que poderão contribuir para a evolução do SDO. As principais delas são: 1) Realização de novas avaliações com especialistas oftalmológicos e usuários para testar cenários de uso padrão do aplicativo e identificar possíveis melhorias; 2) Refinamento dos filtros, além da adição de filtros adicionais ou opções de personalização aos existentes; 3) Otimização do aplicativo para melhorar a qualidade da experiência de usuário (ie., qualidade da simulação, performance e número de dispositivos compatíveis); 4) Evolução da funcionalidade de maquete $3 \mathrm{D}$ do olho humano.

Em 2020, um projeto de mestrado também foi iniciado com o intuito de investigar o uso do SDO no ciclo de avaliações de acessibilidade de aplicativos e jogos móveis. Em um primeiro momento, os filtros de daltonismos seriam empregados durante o processo de avaliação heurística para identificar problemas de acessibilidade relacionados às escolhas das cores e a outros elementos das interfaces. A previsão de finalização é março de 2022.

\section{CONSIDERAÇÕES FINAIS}

No seu estado atual, o produto desenvolvido já possui um nicho como ferramenta para auxílio na realização de testes e simulações das patologias. Foram desenvolvidos 4 filtros, cada um representando uma das doenças oculares mais prominentes na população mundial, que podem ser facilmente personalizados e trocados em tempo real, seja pela interface do próprio aplicativo ou por um controle Bluetooth. Devido aos baixos requisitos de hardware e suporte mínimo para versão 19 do Android SDK (correspondente ao Android OS 4.4, KitKat), o aplicativo pode ser instalado na maioria dos dispositivos Android atuais atualmente [17]. Assim, o simulador promove uma forma rápida e prática de testar os efeitos do Glaucoma, da Catarata, da DMRI e de múltiplos tipos de Daltonismo sem necessidade de ambiente específico ou dependência a equipamentos externos.

Este trabalho proporcionou, também, uma ótima oportunidade para explorar diversos recursos do motor de jogo Unity e compreender melhor o funcionamento de motores de jogos de modo geral. Foram abordados tópicos relacionados a múltiplos aspectos do ciclo de desenvolvimento de software como performance, modularização, desenvolvimento multiplataforma e future-proofing, além de UI/UX, acessibilidade e usabilidade. Contribuiu, também, para o aprendizado do time de desenvolvimento, em relação a tópicos de tratamento e prevenção de doenças visuais e das patologias existentes, além de detalhes das dificuldades vividas pelos pacientes.

\section{REFERÊNCIAS}

[1] Chadia Abras, Diane Maloney-Krichmar, and Jenny Preece. 2004. User-centered design. Bainbridge, W. Encyclopedia of Human-Computer Interaction. Thousand Oaks: Sage Publications 37, 4 (2004), 445-456.

[2] American Optometric Association. 2020. Macular Degeneration. https://www.aoa.org/healthy-eyes/eye-and-vision-conditions/maculardegeneration?sso=y [Acesso em 8/10/2020].

[3] Halim Cagri Ates, Alexander Fiannaca, and Eelke Folmer. 2015. Immersive simulation of visual impairments using a wearable see-through display. In Proceedings of the Ninth International Conference on Tangible, Embedded, and Embodied Interaction. 225-228.

[4] Ronald T Azuma. 1997. A survey of augmented reality. Presence: Teleoperators \& Virtual Environments 6, 4 (1997), 355-385.

[5] D Banks and RJ McCrindle. 2008. Visual eye disease simulator. Proc. 7th ICDVRAT with ArtAbilitation, Maia, Portugal (2008).

[6] Doug A Bowman and Ryan P McMahan. 2007. Virtual reality: how much immersion is enough? Computer 40, 7 (2007), 36-43.

[7] Kenny Tsu Wei Choo, Rajesh Krishna Balan, and Youngki Lee. 2019. Examining Augmented Virtuality Impairment Simulation for Mobile App Accessibility Design. In Proceedings of the 2019 CHI Conference on Human Factors in Computing Systems. 1-11.

[8] Dr. 2020. Catarata | Drauzio Varella. Drauzio Varella (Aug 2020). https:// drauziovarella.uol.com.br/doencas-e-sintomas/catarata [Acesso em 9/10/2020].

[9] John D Gould and Clayton Lewis. 1985. Designing for usability: key principles and what designers think. Commun. ACM 28, 3 (1985), 300-311.

[10] IanPudney. 2020. Colorblind. https://github.com/IanPudney/Colorblind [Acesso em $9 / 10 / 2020]$.

[11] Ibge. 2020. IBGE | Biblioteca | Detalhes | Acesso à internet e à televisão e posse de telefone móvel celular para uso pessoal : 2017. https://biblioteca.ibge. gov.br/index.php/biblioteca-catalogo?view=detalhes\&id=2101631 [Acesso em 18/09/2019].

[12] Ibgeeduca. 2019. IBGE - Educa | Jovens. https://educa.ibge.gov.br/jovens/ conheca-o-brasil/populacao/20551-pessoas-com-deficiencia.html [Acesso em 18/09/2019].

[13] National Eye Institute. 2019. Cataracts | National Eye Institute. https://www.nei. nih.gov/learn-about-eye-health/eye-conditions-and-diseases/cataracts [Acesso em $9 / 10 / 2020]$.

[14] National Eye Institute. 2020. Facts About Color Blindness | National Eye Institute. https://web.archive.org/web/20160728003639/https://nei.nih.gov/health/ color_blindness/facts_about [Arquivado. Acesso em 16/10/2020].

[15] National Eye Institute. 2020. Glaucoma | National Eye Institute. https://www.nei. nih.gov/learn-about-eye-health/eye-conditions-and-diseases/glaucoma [Acesso em $9 / 10 / 2020]$

[16] James Lewis, David Brown, Wayne Cranton, and Robert Mason. 2011. Simulating visual impairments using the Unreal Engine 3 game engine. In 2011 IEEE 1st International Conference on Serious Games and Applications for Health (SeGAH). IEEE, $1-8$.

[17] StatCounter Global Stats. 2020. Mobile Android Version Market Share Worldwide | StatCounter Global Stats. https://gs.statcounter.com/android-version-marketshare/mobile/worldwide [Acesso 12/10/2020].

[18] World Health Organization: Who. 2019. Disability. World Health Organization: WHO (Jan 2019). https://www.who.int/health-topics/disability\#tab=tab_1

[19] World Health Organization: Who. 2020. Blindness and vision impairment. World Health Organization: WHO (Oct 2020). https://www.who.int/en/news-room/factsheets/detail/blindness-and-visual-impairment [Acesso em 9/10/2020]. 